\title{
Cardiovascular Pathology in Women Who Received Radioactive Iodine Exposure During Pregnancy as a Result of the Chernobyl Accident
}

\author{
Stojarov AN ${ }^{1 *}$, Khrustalev VV ${ }^{2}$ and Adzintsova-Stazharava $\mathrm{DA}^{3}$ \\ ${ }^{1}$ Department of Radiation Medicine and Ecology, Belarusian State Medical University, Minsk, Belarus \\ ${ }^{2}$ Department of General Chemistry, Belarusian State Medical University, Minsk, Belarus \\ ${ }^{3}$ Department of Obstetrics and Gynecology, Belarusian State Medical University, Minsk, Belarus \\ *Corresponding author: Stojarov AN, Department of Radiation Medicine and Ecology, Belarusian State Medical University, Belarus
}

ARTICLE INFO

Received: 㓞 July 10, 2021

Published: 幽 July 20, 2021

Citation: Stojarov AN, Khrustalev VV, Adzintsova-Stazharava DA. Cardiovascular Pathology in Women Who Received Radioactive Iodine Exposure During Pregnancy as a Result of the Chernobyl Accident. Biomed J Sci \& Tech Res 37(2)2021. BJSTR. MS.ID.005984.

Keywords: I-131; Accident at the Chernobyl Nuclear Power Plant; Cardiovascular Disease; Absorbed Dose

Abbreviations: CVD: Cardiovascular Diseases; TG: Thyroid gland; CVS: Cardiovascular System; PTSD: Post-Traumatic Stress Disorder; DSM-IV: Diagnosis of Mental Illness; TSH: Thyroid-Stimulating Hormone

\begin{abstract}
The morbidity structure of pregnant women (main cohort) irradiated due to the iodine incorporation differs from women who were not irradiated, but pregnant at a later date after the Chernobyl accident (control cohort). The incidence of cardiovascular diseases (CVD) in the main cohort of women began to occur immediately after the accident, while in women from the control cohort the beginning of the registration of this pathology was shifted to a later period (1999). The morbidity in the main cohort is higher than that in the control group from 1986 until 2008, after which the morbidity is higher in the control cohort, i.e. among women who have not been injured by iodine during pregnancy. The maximum incidence of all types of CVD in pregnant women who were exposed to radioactive iodine in April 1986 was in 2011. The incidence curve for women who were not irradiated during pregnancy shows one broad peak centered in the 2008 region. These data can be explained by the influence of the psycho-emotional consequences of the Chernobyl accident on the general somatic morbidity.
\end{abstract}

On the diagram of the dependence of morbidity on the absorbed dose by the thyroid gland, two peaks are clearly revealed. The first of them, that is wide, corresponds to the absorbed dose by the thyroid gland equal to $70 \mathrm{mGy}$. The second, that is very narrow one, corresponds to the absorbed dose equal to $160 \mathrm{mGy}$. The highest incidence rates (19862016) were typical for women exposed to radiation in the second trimester of pregnancy. The obtained data can be explained by the instability of specific genes of the thyroid gland of pregnant women after the incorporation of I-131, which can be responsible for the synthesis of thyroid hormones, and the level of the latter can be reflected in the appearance of cardiovascular pathology.

\section{Introduction}

It is well known that the growth of the incidence of oncological diseases in the irradiated population is associated with the effect of ionizing radiation. This was especially evident after the accident at the Chernobyl nuclear power plant. As a result of the release of a large amount of radioactive iodine into the environment, the incidence of thyroid cancer in children began to increase within 5 years in Belarus [1], and then the incidence of this oncopathology has begun to increase among adults as well [2]. Meanwhile, it is very interesting to clarify the question of whether radiation exposure is associated with non-oncological, i.e. general somatic pathology. For a long time, this connection was denied or correlations were found in case of relatively high doses of radiation only [3]. Such studies were carried out on victims of the atomic bombings in Hiroshima and Nagasaki [4]. These studies indicated the existence of a certain relationship between population exposure and growth of non-cancer morbidity in patients [5]. Particularly relevant is the 
issue about human exposure to low doses of radiation, within a few hundred mGy or tens of mSv.

This issue is also a priority in prospective studies of the United Nations Scientific Committee on the Effects of Atomic Radiation (UNSCEAR). It is necessary to find out which organs and systems of the human body can be exposed to small doses of radiation and what non-oncological pathology may subsequently take place. This kind of research is important for all groups of the population, while pregnant women represent a special group. The fact is that this period of life is characterized by special functional features. The hormonal background changes sharply, the function of many body systems is being rebuilt to maintain homeostasis and the functions of the developing child's body. All those changes are accompanied by a significant restructuring of the mother's body. During this period of time, pregnant women can be much more sensitive to the effects of environmental factors, including radiation. The period of pregnancy is accompanied by complex changes in the function of the thyroid gland (TG), which can affect the body of the fetus, and subsequently the child. The organism of mother herself cannot be left aside. In the literature, there are very scanty data on the effect of the radiation factor on pregnant women.

The purpose of this work was to analyze the incidence of cardiovascular diseases (CVD) in women over the past 30 years after the Chernobyl accident. Only those women who were pregnant in April 1986 and as a result of the accident received doses to the thyroid gland due to the incorporation of I-131 and then continued to live in territories contaminated by radionuclides were included in this study.

\section{Materials and Methods}

The cohort of women who received radiation as a result of the Chernobyl accident (April-May 1986) and who were during this period at various stages of pregnancy was formed according to the data of the local authorities of the Stolin district of the Brest region, obtained by direct measurements of the exposure dose rate (April-June 1986) over the area of the thyroid gland in various categories of the population. The search for those persons and their identification was carried out in the databases of the State Register of persons affected by the Chernobyl accident, which had medical documentation. We designated this group as the main one. A total of 221 women were included in it. The calculated absorbed doses by the thyroid gland due to radioactive iodine (I-131) were provided by the head of the laboratory for reconstructing radiation doses to the population of the State Research Center of the Federal Medical Biophysical Center named after V.I. A.I. Burnazyan FMBA of Russia Doctor of Technical Sciences, Shinkarev S.M. Doses were calculated using a semi-empirical 2004 iteration model. In addition, for some cases, the doses absorbed by the thyroid gland were calculated according to the data of radiometric, so-called "direct" measurements of the exposure dose rate over the area of this organ location, carried out from April 26 to June 30, 1986 [6].

Since pregnant women after the "iodine strike" (exposure to I-131) continued to live in the area contaminated with radionuclides, the analysis also took into account the effective doses from radioactive Cs-137, which contaminated the area. The indicated total doses (1986-2016) were calculated using the RESRAD-ONSITE software, version 7.2 (Enviromental Science Division of Argonne National Laboratory, USA). The density of the surface activity of the soil of the Stolin district for Cesium-137 was provided by the State Institution "Republican Center for Hydrometeorology, Control of Radioactive Contamination and Environmental Monitoring”. In the structure of the total dose, external, inhalational and internal irradiation was taken into account. The diet included: whole milk products - $100 \mathrm{~kg}$, meat products - $91 \mathrm{~kg}$, plant products - $163 \mathrm{~kg}$.

A cohort for comparison included women from the same Stolin district of the Brest region, whose pregnancy occurred later, i.e. in 1987. Due to the short half-life of I-131 (8 days) after a year, i.e. in 1987, there was almost no radioactive iodine in the environment and it did not affect pregnant women. However, the same living conditions on the territory of the Stolin district, the same radiation exposure from the contaminated area, their social status and age served as good conditions for choosing them as a control group. The cohort for comparison included 40 women. The morbidity data were obtained from the State Register of Persons Affected by the Chernobyl Accident. The work took into account only the primary morbidity. Statistical data processing was carried out using the applied computer programs Statistics 10.0 (StatSoft. Inc, USA) and SigmaPlot 12.5 (Systat Software Inc., Germany). This study was approved by the Ethics Commission of the Belarusian Association of Physicians (10.12.2020).

\section{Results}

Figure 1 shows the incidence of diseases of the cardiovascular system (CVS) in a cohort of women whose thyroid gland incorporated radioactive iodine (I-131) during pregnancy. In the first place among all pathologies there is essential (primary) hypertension (I10 - 33.0\%). Atherosclerotic heart disease was approximately two times less common (I25.1 - 17.7\%). Chronic ischemic disease (I25) and hypertensive heart disease (with predominant heart disease) without (congestive) heart failure (I11.9) occurred in women with the same frequency of $4.2 \%$ and $2.3 \%$, respectively. Other types of pathology were significantly less pronounced, such as various forms of angina pectoris, as well as of vascular diseases. A completely different picture of the morbidity structure was observed in the control group, i.e. in women who were pregnant later and did not fall under the "iodine strike" at the end of April-May 1986 (Figure 2). As stated earlier, by this time I-131 had practically completely decayed. 


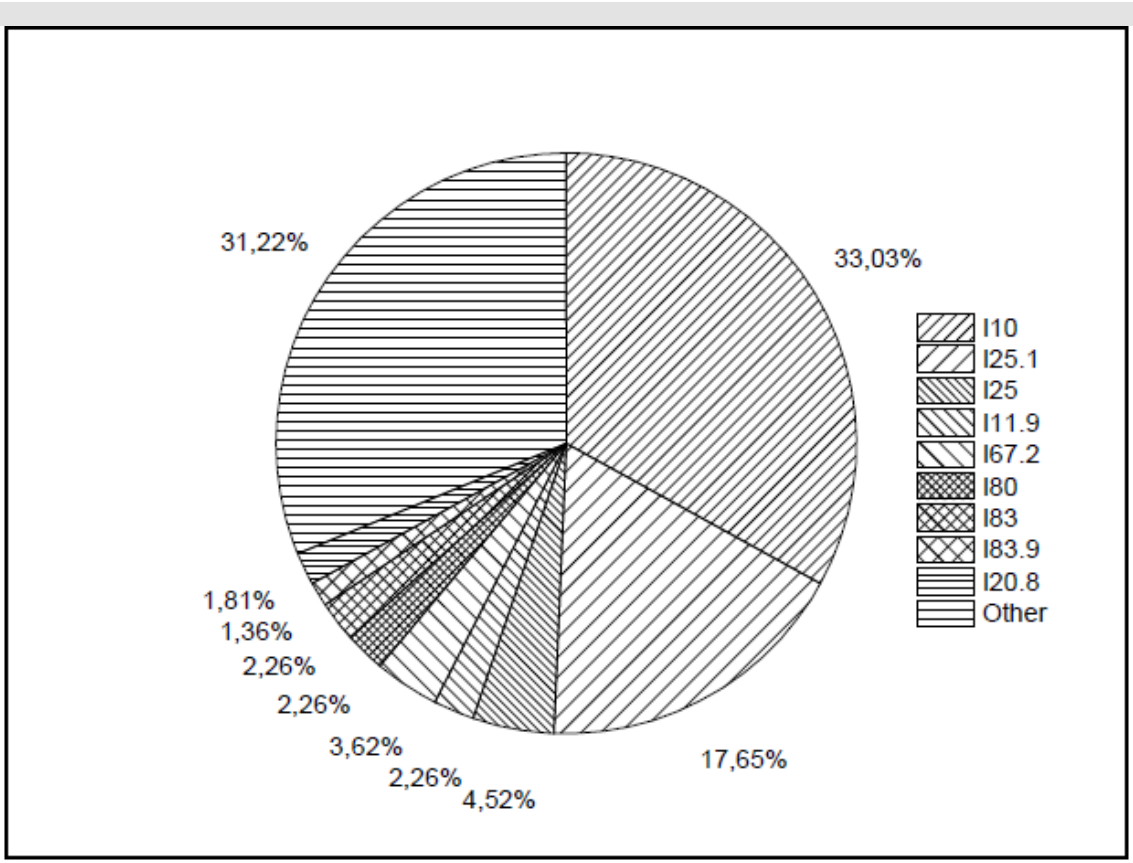

Figure 1: The incidence of diseases in the cohort of women who received exposure to radioactive iodine during pregnancy.

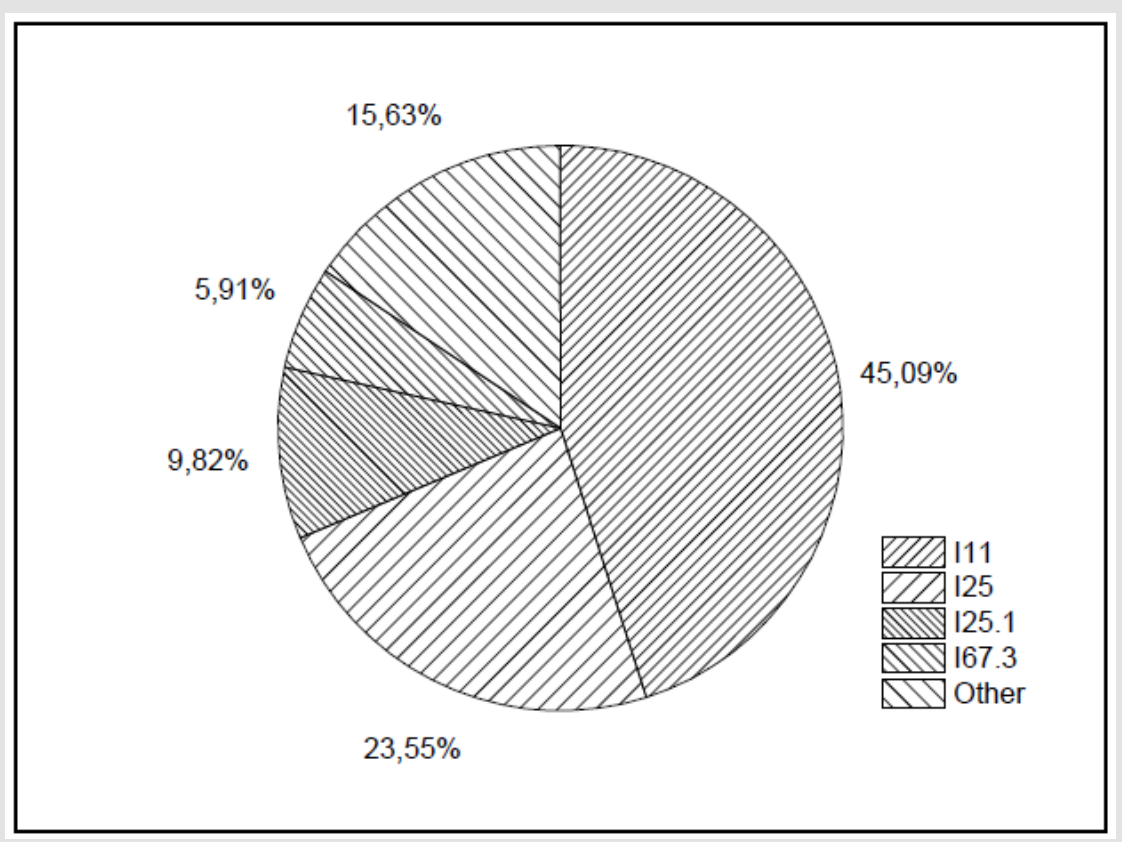

Figure 2: The pattern of morbidity in a cohort of women who were not irradiated by radioactive iodine during pregnancy.

It is clearly seen (Figure 2) that hypertensive heart disease dominates (I11 - 45.1\%). The second place in the structure of morbidity belongs to chronic ischemic (I25) and atherosclerotic heart diseases (I25.1). Essential hypertension was registered in the cohort for comparison only once ( $2 \%$ of all cases). The study of the cumulative morbidity during the entire studied time interval showed that the incidence of CVD in the cohort of irradiated women began to be registered immediately after the accident (Fig. 3), while in the cohort of non-irradiated women the beginning of the registration of diseases was shifted to a later period (1999). The morbidity in the cohort of women irradiated during their pregnancy was higher than that in women who were not irradiated during their pregnancy until 2008 (Figure 3), after which an increased incidence of cardiovascular diseases began to be recorded in the cohort of women who did not fall under the "iodine blow" during pregnancy. 


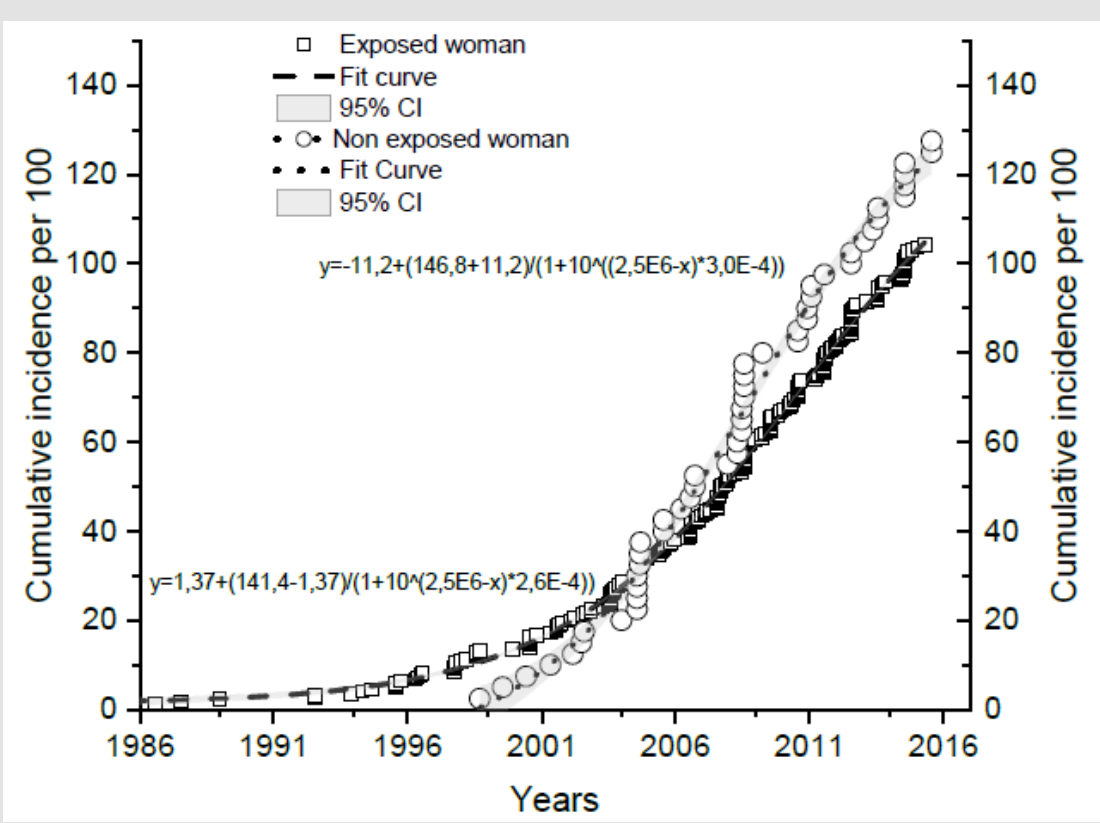

Figure 3: Cumulative morbidity of all forms of cardiovascular diseases in cohorts of women irradiated and non-exposed to radiation during their pregnancy.

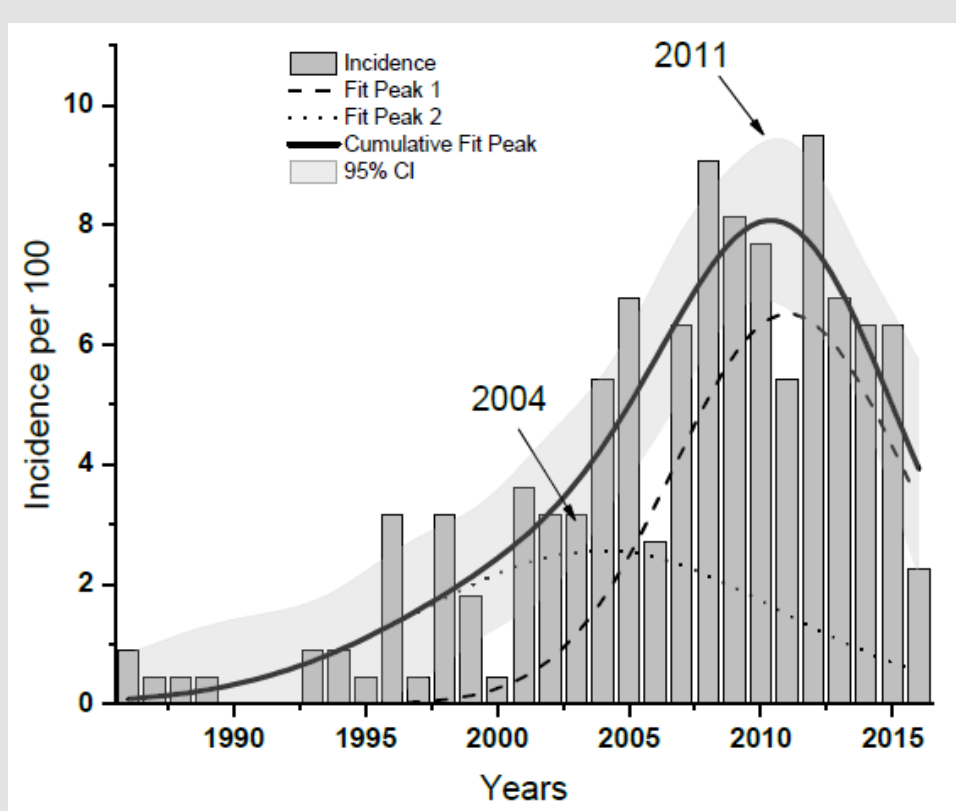

Figure 4: Incidence of CVD (from 1986 to 2016) among women who were pregnant during the Chernobyl accident and were irradiated by I-131.

Analysis of the data showed that the incidence of all types of CVD in pregnant women who were exposed to radioactive iodine in April 1986 has a maximum in 2011, although this dome-shaped relationship is asymmetric due to the flattening of the left shoulder of the peak, which may be due to the superposition of a small maximum detected in the 2004 (Figure 4). Registration of the time dependence of morbidity in women who were not exposed to radioactive iodine revealed a slightly different dependence (Figure 5). There is a broad peak on the incidence curve centered in the
2008 region. As in the previous case, an additional peak around the year 2004 is also revealed on the left shoulder of the function of dome-shaped dependence. At the same time, it is clearly noticeable that until 1990, in this group of women, in contrast to those affected by the "iodine stroke", the incidence of CVD was not recorded. These data are in good agreement with the results shown in Figure 3 , which also clearly shows that the onset of morbidity is different in both groups. 


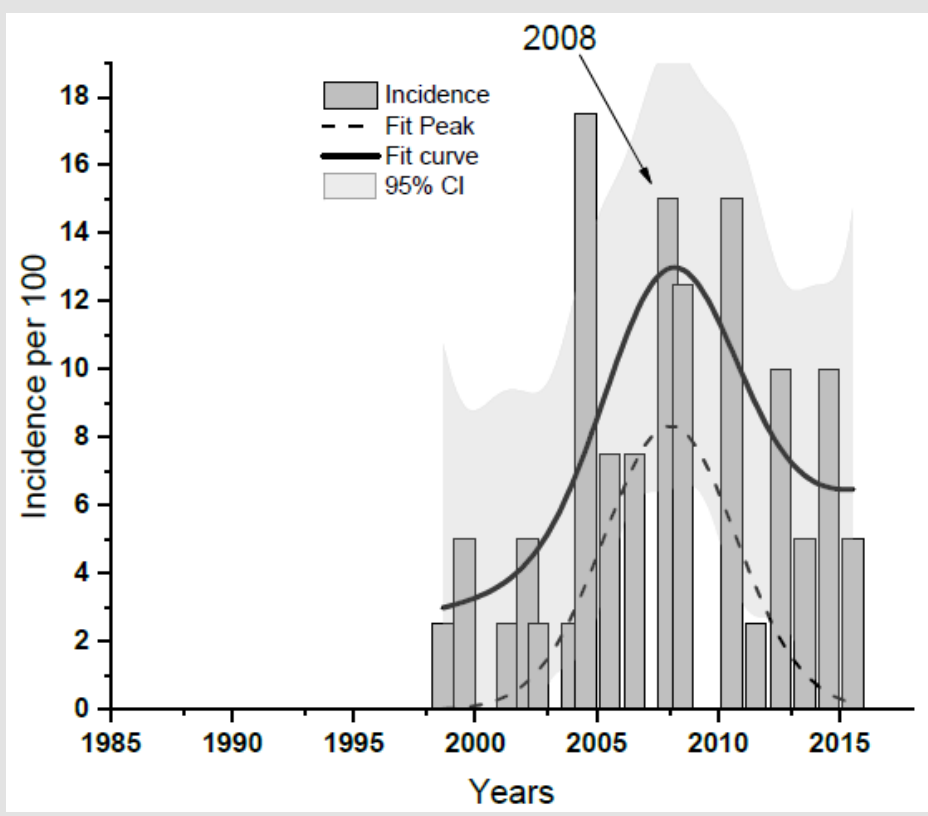

Figure 5: The incidence of CVD (from 1986 to 2016) among women who were not pregnant during the Chernobyl accident but were irradiated by I-131.

One of the next steps in this work was to identify the dose dependence, i.e. to compare the incidence of CVD with doses absorbed by the thyroid gland of pregnant women due to the incorporation of radioactive iodine. Figure 6 shows the cumulative incidence of CVD in the cohort of women who were exposed during their pregnancy. Attention is drawn to the absence of any relationship between these two indicators. Cardiovascular pathology occurred in the range of absorbed doses by the thyroid gland of women of 50-120 and 160-250 mGy. Up to $50 \mathrm{mGy}$, as well as in the range of 120 - $155 \mathrm{mGy}$, no pathology was found in the future. The same data, but presented in different coordinates, showed approximately the same dependence. The dose dependence diagram clearly shows two peaks (Figure 7). The first of them, that is wide, corresponds to the absorbed dose by the thyroid gland in the region of $70 \mathrm{mGy}$. The second, that is very narrow, is in the region of the absorbed dose equal to $160 \mathrm{mGy}$. These data are unexpected and therefore require careful evaluation.

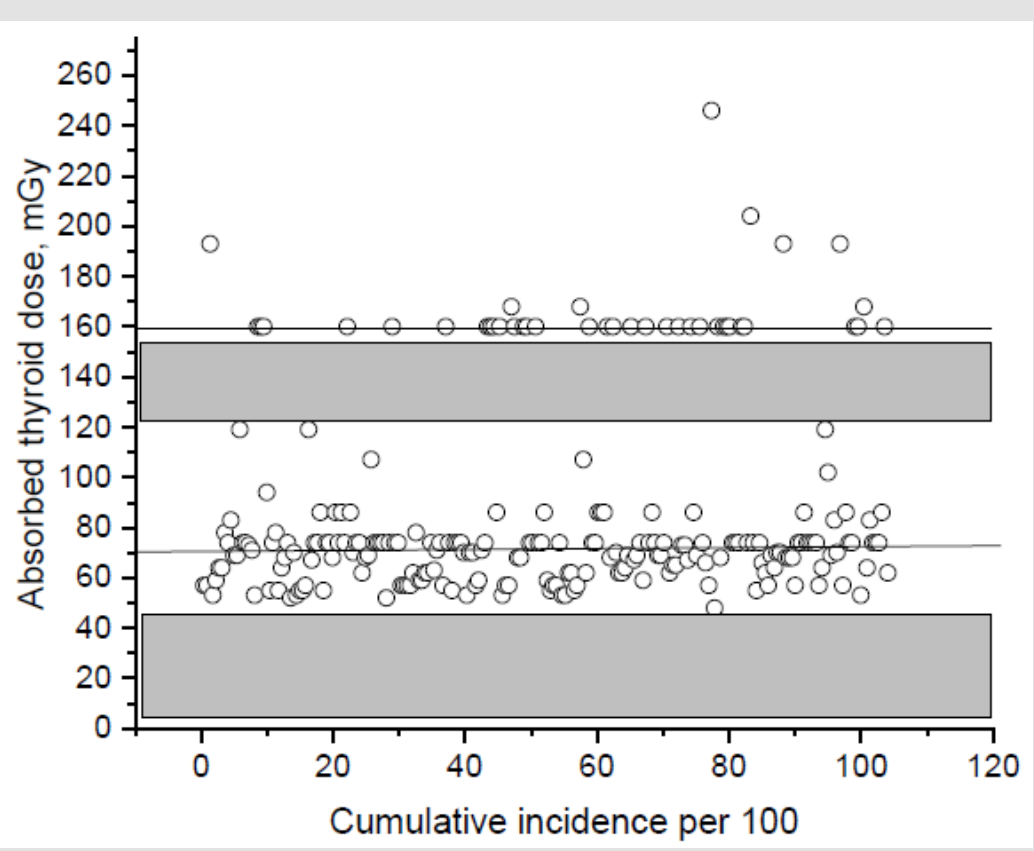

Figure 6: Cumulative incidence of CVD in the cohort of irradiated pregnant women (1986-2016). 


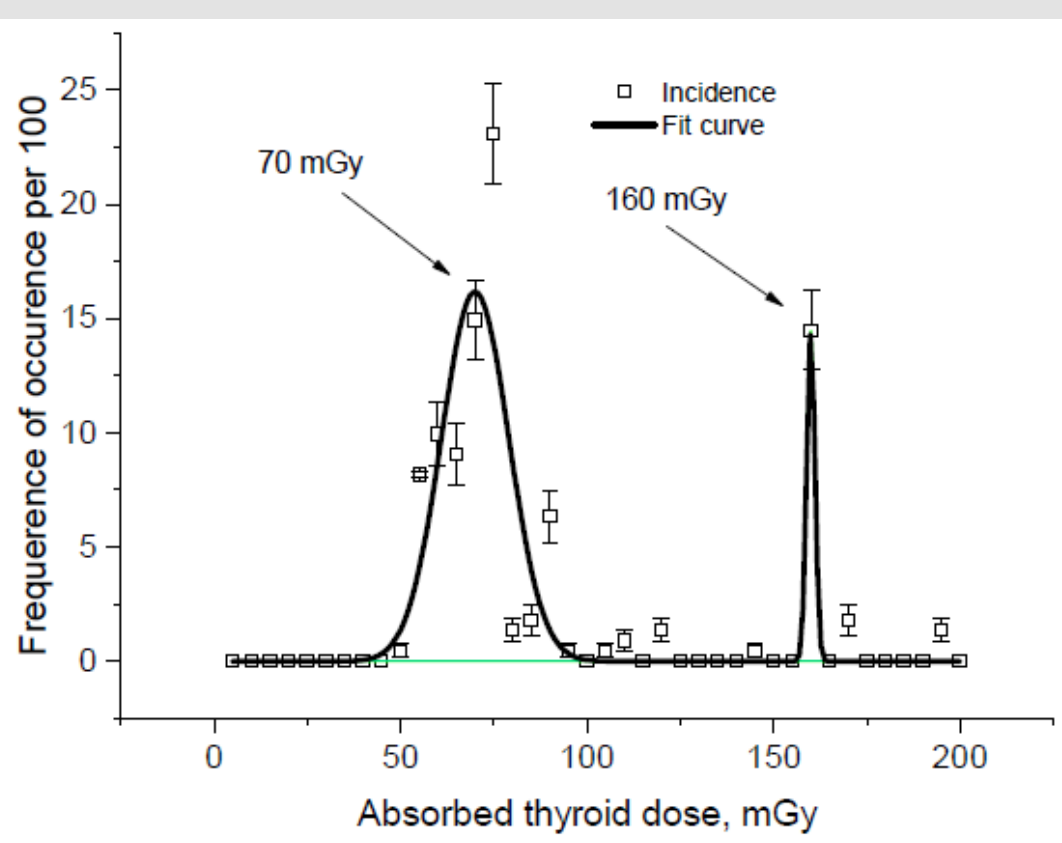

Figure 7: Dose dependence of the incidence of CVD in the group of pregnant women irradiated during the pregnancy. The figure shows data with an error of the indicator.

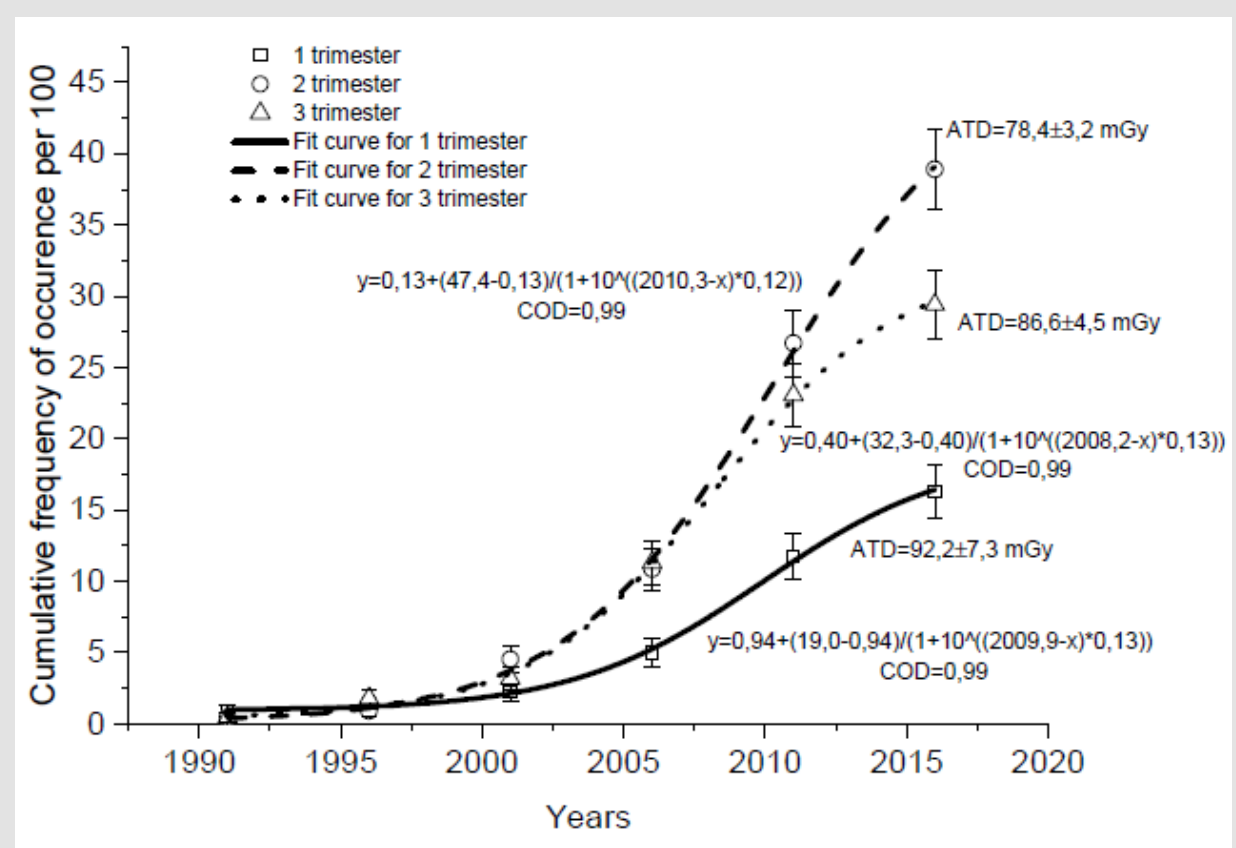

Figure 8: Cumulative incidence of CVD in women exposed to radiation at different trimesters of pregnancy. The figure shows data with an error of the indicator.

Note: ATD - absorbed thyroid dose (mGy).

It is well known that during pregnancy, certain periods (trimesters) should be distinguished during which the functional characteristics of the woman's body, her hormonal background, adaptive and reserve reactions change. Also, significant changes in the fetal organism occur: the laying of organs, their development and differentiation, etc. In this regard, it was of interest to analyze the timing of pregnancy, during which the irradiation happened, for the subsequent morbidity of women. The first trimester corresponds to $1-12$ weeks, the second trimester corresponds to 13-26 weeks and the third trimester corresponds to 27-41 weeks of 
pregnancy. In Figure 8 the data on the incidence of CVD, depending on the period of pregnancy during which the irradiation by I-131 happened, are presented. It was found that irradiation of women in the first trimester of pregnancy was accompanied by the lowest incidence of CVD. The average absorbed dose by the thyroid gland in this group was $92 \mathrm{mGy}$. The highest values of the frequency of occurrence were typical for irradiation of women in the second trimester (the absorbed dose was $78 \mathrm{mGy}$ ). Irradiation in the third semester of pregnancy was characterized by intermediate values of the incidence of CVD (absorbed dose was 87 mGy).

\section{Discussion}

The analysis of the presented data is of the undoubted interest. First, the fact of an increase in the incidence of CVD in the postaccident period in both cohorts of women requires explanation. The increase in the incidence of cardiovascular pathology in the main cohort began to be recorded immediately after the accident (Figure 3 ). In the cohort of women who were not irradiated exactly during the pregnancy, this pathology began to occur much later, starting from 1999. After some period of time (after 2008), the incidence of CVD in the control cohort began to exceed the same indicator in the main cohort. Living conditions of the mentioned cohorts of women were the same, and so they received approximately the same exposure. It can be assumed that in the main cohort of women, the role of an inducer of the growth of cardiovascular pathology could have been played by the irradiation of the thyroid gland during the "iodine stroke" in April-May 1986 when they were pregnant. There is an evidence that low-dose irradiation is associated with the occurrence of hypothyroidism [7], and changes in the level of thyroid hormones can have a significant effect on cardiovascular hemodynamics [8].

However, in women who were pregnant at a later date after the Chernobyl accident the incidence of CVD is also growing in time. A possible explanation for this fact can be found in our previous studies that included a cohort of women who were not irradiated at all. Studying the psycho-emotional status of the same cohort of irradiated women due to the incorporation of radioactive iodine and non-irradiated pregnant women, we were able to establish that a cohort of women exposed to radiation is characterized by the absence of a clear connection between post-traumatic stress disorder (PTSD) and psychological distress. At the same time, only the "Avoidance" factor according to the five-factor model of guidance in the diagnosis of mental illness (DSM-IV), which was analyzed using the PCL-S questionnaire in a group of women over the age of 19 with stress indicators, was especially clearly manifested [9]. This can be the reason for the smoothness of the emotional state, i.e. avoidance of experiences after 30 years after the Chernobyl accident.

A psychometric analysis of women whose pregnancy occurred a year after the Chernobyl accident, on the contrary, showed a strong correlation between most of the factors of PTSD with psychological distress (factors of "Re-experiencing", "Numbing", "Dysphoric Arousal" and "Anxious Arousal" excitements) [10]. These signs were expressed in sleep disturbances, insomnia, anxious alertness, etc. It is known that the period after the Chernobyl accident was characterized by an abundance of often inaccurate information about the effects of radiation. Fear for the health of the unborn child and of the own health could play an important role in the development of latent internal tension in women. There is an evidence that an altered psychological status accompanied by the stress can be the cause of the subsequent appearance of somatic pathology [11]. In this regard, the delayed growth of CVD pathology in the mentioned cohort of women who had been irradiated, but became pregnant later, becomes clear.

The maximal values of the incidence rates in the main (2011) and control (2008) cohorts can be explained by the onset of menopause in women. Since the average age of women in the main cohort in April 1986 was 24 years old, and the average age of women in the control cohort was 26 years old, they were over 48 years old at the time indicated above. This is the age of menopause. During this period, the level of estrogens in women decreases, and estrogens are known to serve as protectors of normal function of CVS [12]. So, at the age of menopause numerous complaints of a different nature, including CVD, occur, and women turn to medical specialists with complaints about CVS for the first time. It is known that the early-onset menopause happens in women at the age of 40 - 45 years. The percent of such cases in population is around $10 \%$ [12]. So, the hidden peak at 2004 year from Figure 4 might occur because of women with early-onset menopause that is usually more frequent among rural residents, than in those who live in cities.

New data on the peak dependence of the incidence of CVD depending on the absorbed dose by the thyroid gland of pregnant women are of a great interest. As shown in Figure 7, there are two incidence peaks: in the region of 70 and 160 mGy. Recently, convincing evidence has been obtained that radiation damage, in addition to the effect on the genetic apparatus of the cell, causes a complicated cascade of biochemical processes associated with the involvement of extra- and intracellular signaling systems, anti-inflammatory cascade mechanisms of cytokines, etc. [13]. The delayed effects of increased CVD morbidity observed by us in women, many years after the irradiation, can hardly be explained by metabolic changes in thyrocytes. Most likely, they can be associated with the mechanisms of gene expression in these cells. Earlier, in the study of the action of I-131, 27 up- and downregulated genes were found in thyroid cells [14]. Some of them may be of a particular interest in terms of the explanation of changes we found.

Thus, the expression of the Mb gene encoding the MYG protein is suppressed by low doses of radioactive iodine, and sharply increases with at higher doses. MYG is responsible for facilitating 
the transfer of oxygen from the cell membrane to the mitochondria. This protein also plays a role in regulating the physiological levels of nitric oxide. For this gene, there are many variants of transcripts encoding different isoforms. The phosphorylating capacity of mitochondria will play an important role in the production of ATP and, therefore, support the contractile function of the muscles. A number of other genes (Pax8, Sic5a5, Tg, Tpo), which play an important role in the functioning of the thyroid gland, the synthesis of thyroid hormones and the effect on the metabolism of peripheral cells, are downregulated by small doses formed from I-131 and change their activity with an increase of radiation exposure. There are other examples. Thus, the mentioned gene Sic5a5 is also downregulated at low and medium doses of radiation caused by I-131, and it is upregulated at high doses of exposure [14].

From this point of view, the above written data on the existence of two peaks in the incidence of CVD among pregnant women who were irradiated with radioactive iodine in April-May 1986 can be explained. In women, various absorbed doses were formed, which could be responsible for the change in the expression of genes and the appearance of two peaks. The thyroid gland, in turn, plays an important role in the functioning of the cardiovascular system through the production of its hormones [15]. At the same time, it is known that ionizing radiation induces genome instability [16]. The consequences of radiation-induced genome instability are: morphological and functional inferiority of tissue elements at the tissue level, the increased risk of developing tumors and non-tumor somatic pathology at the organismal level. Genome instability is a persistent, epigenetically transmitted, functional state of the cell genome, leading to a violation of genetic control and being the most important factor in the induction of pathology. These changes can be fixed in the body of pregnant women, and therefore we observe these effects many years after the Chernobyl accident.

It is known that certain periods of pregnancy are associated with complex changes in the pituitary-thyroid system of women [17]. In different trimesters of pregnancy, many biochemical indicators change, including the levels of T4, T3, thyroidstimulating hormone (TSH), etc. So, at the end of the first trimester of pregnancy (at 10 weeks), the TSH level drops to minimum values, and then, as the gestation period increases (by week 20), its level is coming back to a normal value [18]. Accordingly, the T4 level in the woman's body will also change. For the formation of T4, the transmembrane transport of iodide (I-) is required, first through the basolateral and then through the apical membranes into the colloid for the subsequent iodination of thyroglobulin. For these processes, the expression of the Sic5a5 / NIS, Tg genes, and possibly other genes is required [14]. It has already been mentioned that their expression demonstrates a dose dependence. The inhibitory effect is pronounced at lower doses of I-131, and at higher doses it decreases.
According to our observations, irradiation in the second trimester of pregnancy was accompanied by the formation of lower absorbed doses by the thyroid gland of women (Figure 7). Against the background of a decrease in the level of TSH, an insufficient level of transmembrane transfer of iodide will negatively affect the state of the thyroid system of a woman and a fetus and, due to the mentioned instability of the genome, may serve as the reason for the subsequent appearance of non-oncological pathology. However, it should be kept in mind that the mechanisms suggested by us should work only in pregnant women, due to the fact that this physiological state is characterized by the tension of many body systems, including endocrine, and can be the cause of genome instability under radiation exposure.

\section{Conclusion}

The incidence of CVD in the cohort of women irradiated during the pregnancy began to be registered immediately after the accident, while in the group of women who were irradiated but not pregnant at that time, the beginning of the registration of the onset of CDV was shifted to a later period (1999) and after 2008 began to exceed that in the first cohort. These data can be explained by the peculiarities of the psycho-emotional state of women in the control cohort, who became pregnant soon after the Chernobyl accident: signs of stress due to the abundance of information about radiation, which are capable of inducing general somatic pathology. On the diagram of the dependence of morbidity on the absorbed dose by the thyroid gland, two peaks are clearly evident. The first of them, that is wide, corresponds to the absorbed dose by the thyroid gland in the region of $70 \mathrm{mGy}$. The second, that is very narrow one, exists in the region of the absorbed dose equal to $160 \mathrm{mGy}$. The highest CVD incidence rates were typical for women exposed to radiation in the second trimester. The obtained data can be explained by the instability of expression of specific genes of the thyroid gland in the organism of pregnant women after the incorporation of I-131, which are capable of being responsible for the synthesis of thyroid hormones, and other functions, which will cause the appearance of cardiovascular pathology.

\section{References}

1. Ron E (2007) Thyroid Cancer Incidence Among People Living in Areas Contaminated by Radiation From the Chernobyl Accident. Health Phys 93(5): 502-511.

2. Stojarov AN, Okeanov AE, Khrustalev VV, Alesia AY (2021) Thyroid cancer in persons as a result of the Chernobyl Accident. Instant Journal of Hematology and Oncology 2: 1-11.

3. Gilliesa M, Richardson D, Cardis E, Daniels RD, Hagan JAO, et al. (2017) Mortality from Circulatory Diseases and other Non-Cancer Outcomes among Nuclear Workers in France, the United Kingdom and the United States (INWORKS) Radiat Res 188(3): 276-290.

4. ShimizuY, Kodama K, Nishi N, Kasagi F, Suyama A, et al. (2010) Radiation exposure and circulatory disease risk: Hiroshima and Nagasaki atomic bomb survivor data, 1950-2003. BMJ 1: 1-8. 
5. Report of United Nations Scientific Committee on the Effect of Atomic Radiation. 2010, Fifty-seventh session, includes Scientific Report: summary of low-dose radiation effect on health, p. 1-14

6. Gavrilin Y, Khrouch V, Shinkarev S, Drozdovitch V, Minenko V, et al. (2004) Individual thyroid dose estimation for a casecontrol study of Chernobylrelated thyroid cancer among children of Belarus-part I: 131I, shortlived radioiodines (132I, 133I, 135I), and short-lived radiotelluriums (131MTe and 132Te). Health Phys 86: 565-585.

7. Ostroumova E, Rozhko A, Hatch M, Furukawa K, Polyanskaya O, et al (2013) Measures of thyroid function among Belarusian children and adolescents exposed to iodine-131 from the accident at the Chernobyl nuclear plant. Environ Health Perspect 121(7): 865-871.

8. Jabbar A, Pingitore A, Pearce S, Zaman A, Iervasi G, et al. (2017) Thyroid hormones and cardiovascular disease. Nat Rev Cardiol 4(1): 39-55.

9. Stozharov AN, HayashidaN, Takahashi J (2017) Psychometric analysis of the data of the questionnaire of the severity of post-traumatic stress in women irradiated during pregnancy as a result of the accident at the Chernobyl nuclear power plant. The materials of the Republican Scientific and Practical Conference with international participation «Health and Environment»: Minsk 1: 95-99.

10. Odintsova-Stojarova D, Mozheiko L, Stozharov A, Weinstein L (2021) Character of psycho-emotional disorders in women irradiated during the accident at the Chernobyl nuclear power plant. Medical Journal 3: 82-86.

ISSN: 2574-1241

DOI: 10.26717/BJSTR.2021.37.005984

Stojarov AN. Biomed J Sci \& Tech Res

(C) This work is licensed under Creative

Submission Link: https://biomedres.us/submit-manuscript.php
11. Blanchard EB, Jones-Alexander J, Buckley NC, Forneris CF (2005) Posttraumatic stress and depressive syndrome in a college population one year after the September 11 attacks: The effect of proximity. Behaviour Research and Therapy 43: 143-150.

12. Muka T, Oliver-Williams C, Kunutsor S, Laven JSE, Bart C J M Fauser, et al. (2016) Association of age at onset of menopause and time since onset of menopause with cardiovascular outcomes, intermediate vascular traits, and all-cause mortality: A systematic review and meta-analysis. JAMA Cardiol 1(7): 767-776.

13. Sally A (2008) Functional genomics in radiation biology: a gateway to cellular systems-level studies. Radiat Environ Biophys 47(1): 25-31.

14. Rudqvist N (2015) Radiobiological effects of the thyroid gland. University of Gothenburg, Gothenburg, p. 69.

15. Navid A, Faisal A, Masehullah S, Ziemnicka K, Minczykowski A (2020) Thyroid gland dysfunction and its effect on the cardiovascular system: a comprehensive review of the literature. Endokrynol Pol 71(5): 466-478.

16. Vorobtsova I (2006) Transgenerative transmission of radiation-induced instability of the genome. Rad Biology R adioecology 46(4): 441-446.

17. Prilutsky A, Glusych S (2015) Physiological changes in thyroid function during pregnancy. International Endocrinological Journal 69(5): 140146.

18. Gaberscek S, Zatelet K (2011) Thyroid physiology and autoimmunity in pregnancy and after delivery. Expert Rev Clin Immunol 7(5): 697-706.

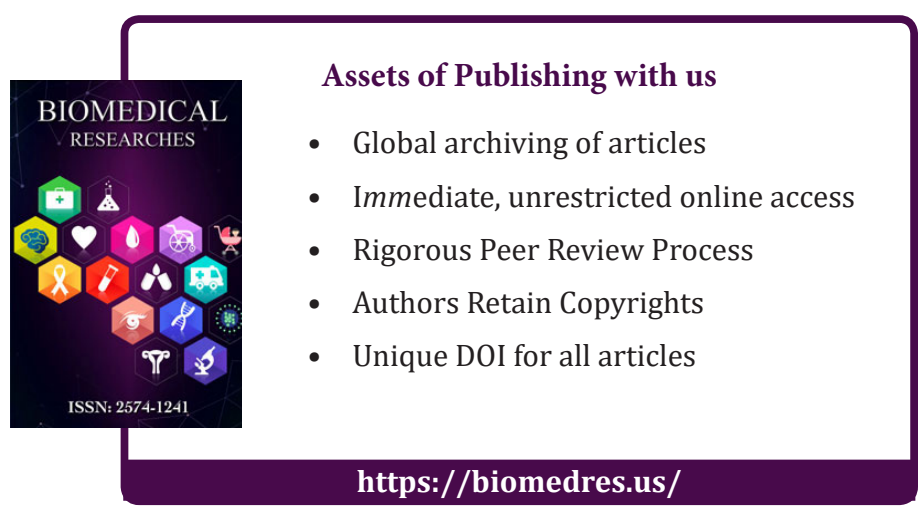

